\title{
Effect of cytokine hemoadsorption on brain death-induced ventricular dysfunction in a porcine model
}

\author{
Krasimira M. Mikhova, BSE, ${ }^{a}$ Creighton W. Don, $\mathrm{MD}, \mathrm{PhD},{ }^{\mathrm{b}}$ Michael Laflamme, MD, PhD, ${ }^{\mathrm{c}}$ \\ John A. Kellum, MD, ${ }^{\mathrm{d}}$ Michael S. Mulligan, MD, ${ }^{\mathrm{a}}$ Edward D. Verrier, MD, ${ }^{\mathrm{a}}$ and David G. Rabkin, $\mathrm{MD}^{\mathrm{a}}$
}

Objective: In an effort to expand the cardiac donor pool, we tested the hypothesis that hemoadsorption of cytokines attenuates brain death-induced ventricular dysfunction.

\begin{abstract}
Methods: Eighteen Yorkshire pigs (50-60 kg) were instrumented with a left ventricular conductance catheter. Cytokine expression, preload recruitable stroke work, and the diastolic relaxation constant tau were measured at baseline and at hourly intervals for 6 hours after induction of brain death by intracranial balloon inflation (brain death, $n=6$ ) or sham operation (control, $n=6$ ). In a third group (brain death +hemoadsorption, $n=6$ ), 3 hours after induction of brain death, animals were placed on an extracorporeal circuit containing a cytokinehemoadsorption device for the remaining 3 hours of the experiment. Myocardial water content was measured after the animals were killed.
\end{abstract}

Results: Six hours after induction of brain death, tumor necrosis factor and interleukin-6 were highest in the brain death group $(106 \pm 13.1 \mathrm{pg} / \mathrm{mL}$ and $301 \pm 181 \mathrm{pg} / \mathrm{mL}$, respectively), lowest in controls $(68.3 \pm$ $8.55 \mathrm{pg} / \mathrm{mL}$ and $37.8 \pm 11 \mathrm{pg} / \mathrm{mL}$, respectively), and intermediate in the brain death + hemoadsorption group $(81.2 \pm 35.2 \mathrm{pg} / \mathrm{mL}$ and $94.6 \pm 20 \mathrm{pg} / \mathrm{mL}$, respectively). Compared with controls, preload recruitable stroke work was significantly reduced in the brain death group 4 hours after the induction of brain death and was $50 \%$ of baseline by 5 hours. In the brain death + hemoadsorption group, preload recruitable stroke work was relatively preserved at $80 \%$ of baseline at similar time points. Tau remained unchanged in the control and brain death + hemoadsorption groups, whereas in the brain death group it was significantly elevated versus baseline $5(139.3 \% \pm 21.5 \%)$ and $6(172 \% \pm 16.1 \%)$ hours after induction of brain death. Myocardial water content was significantly greater in the brain death group than in the other 2 groups.

Conclusions: Hemoadsorption of cytokines using an extracorporeal circuit attenuates brain death-induced ventricular dysfunction in a porcine model. Improvement in function generally correlates with trends in cytokine expression, but this relationship requires further investigation. (J Thorac Cardiovasc Surg 2013;145:215-24)

Although the majority of organs for solid-organ transplants come from cadaveric donors, it has long been recognized that brain death pathophysiology presents a hostile environment to organ function. Brain death has been linked not only to impaired donor organ function, an effect that is particularly pronounced in the heart, ${ }^{1}$ but also to primary graft dysfunction after transplantation ${ }^{2}$ and increased immunogenicity, resulting in a higher

\footnotetext{
From the Divisions of Cardiothoracic Surgery ${ }^{\mathrm{a}}$ and Cardiology, ${ }^{\mathrm{b}}$ and the Department of Pathology, ${ }^{\mathrm{c}}$ University of Washington Medical Center, Seattle, Wash; and the Department of Critical Care Medicine, ${ }^{\mathrm{d}}$ University of Pittsburgh School of Medicine, Pittsburgh, Pa.

Supported in part by the American Surgical Association Foundation Fellowship Award (to D.G.R.), The University of Washington Medical Student Research Training Program (to K.M.M.), and the Department of Surgery at the University of Washington.

Disclosures: Authors have nothing to disclose with regard to commercial support.

Read at the 38th Annual Meeting of The Western Thoracic Surgical Association, Maui, Hawaii, June 27-30, 2012.

Received for publication May 29, 2012; revisions received July 15, 2012; accepted for publication Aug 1, 2012; available ahead of print Nov 5, 2012.

Address for reprints: David G. Rabkin, MD, 1959 NE Pacific St, Room AA115, Box 356310, Seattle, WA 98195 (E-mail: dgr5@u.washington.edu).

$0022-5223 / \$ 36.00$

Copyright (c) 2013 by The American Association for Thoracic Surgery

http://dx.doi.org/10.1016/j.jtcvs.2012.08.002
}

incidence of both acute ${ }^{3}$ and chronic ${ }^{4}$ rejection leading to worse long-term outcomes. Estimates suggest that $20 \%$ of potential donors are excluded from cardiac donation because of the adverse effect of brain death on heart function. ${ }^{5}$ Inspired in part by previous studies that demonstrated that this phenomenon could be reversed in an isolated rat-heart model, ${ }^{6}$ we sought to rehabilitate hearts damaged by the pathophysiology of brain death to expand the donor pool.

Explanations for the mechanism of brain death-induced ventricular dysfunction include ischemic injury, ${ }^{7}$ direct catecholamine-induced myocardial injury, ${ }^{8}$ and impaired B-adrenergic receptor signaling. ${ }^{9}$ Although the phenomenon is likely multifactorial, there is increasing evidence that the surge in inflammatory cytokines that accompanies brain death plays an important role. For example, exogenous cytokine administration has been shown to cause a dose-dependent, reversible injury to ventricular function in both clinical ${ }^{10}$ and experimental settings. ${ }^{11}$ In addition, tumor necrosis factor (TNF) and interleukin (IL)-6 expressions have been shown to be greater in human hearts rejected for transplantation when compared with those accepted for transplantation. ${ }^{12}$ 


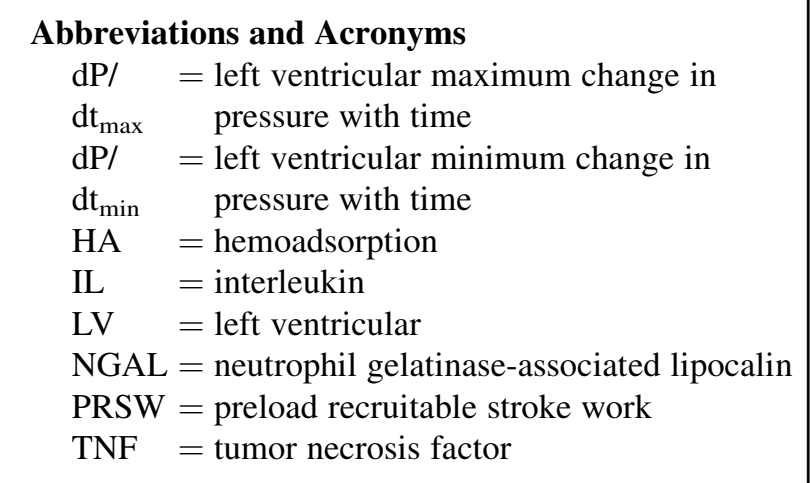

Hemoadsorption (HA) of cytokines using CytoSorb (Cytosorbents Inc, Monmouth Junction, NJ), an adsorption-based cytokine removal device, has been shown to significantly reduce cytokine levels both in vitro ${ }^{13}$ and in septic animals. ${ }^{14}$ In addition, the feasibility of HA in organ donors has been demonstrated. ${ }^{15}$ Therefore, in an effort to address the critical shortage of transplantable hearts, we determined the effect of cytokine HA on brain death-induced ventricular dysfunction.

\section{MATERIALS AND METHODS}

All experiments were approved by the University of Washington Institution for Animal Care and Use Committee and were in compliance with the seventh update of the Guide for the Care and Use of Laboratory Animals, prepared by the Institute of Laboratory Animal Resources and published by the National Institutes of Health (publication no. 85-23, revised 1996, www.nap.edu/catalog/5140.html).

\section{Experimental Model}

Domestic, male Yorkshire-Landrace pigs $(\mathrm{n}=18)($ range, 50-60 kg) were divided into 3 groups. Ventricular function, serum cytokine concentrations, and laboratory indices of pulmonary, renal, and hepatic function were measured at baseline and at hourly intervals, for 6 hours, after induction of brain death (brain death, $n=6$ ) or sham operation (control, $n=6$ ). In the third group (brain death $+\mathrm{HA}, \mathrm{n}=6$ ), 3 hours after induction of brain death, animals were systemically heparinized and subjected to 3 hours of an extracorporeal circuit containing the Cytosorb HA device. Six hours after induction of brain death, hearts were arrested using antegrade crystalloid cardioplegia. Postmortem studies included determination of myocardial water content and histologic stigmata of myocyte injury (Figure 1).

\section{Surgical Preparation}

Animals were premedicated using intramuscular telazol $(2.0-8.0 \mathrm{mg} /$ $\mathrm{kg}$ ) and xylazine $(2.2-4.4 \mathrm{mg} / \mathrm{kg}$ ). They were induced with $3 \%$ to $5 \%$ isoflurane by facemask, endotracheally intubated, maintained on $1 \%$ to $2 \%$ isoflurane, and mechanically ventilated at an initial minute volume of $150 \mathrm{~mL} / \mathrm{kg}$. Adjustments in ventilation parameters were made on the basis of arterial blood gases that were sent periodically to maintain a $\mathrm{pH}$ of 7.35 to 7.45 and a carbon dioxide tension 35 to 45 torr; the fraction of inspired oxygen was kept at 1.0 throughout the experiment. Normothermia was maintained using a Bair Hugger (Arizant Healthcare Inc, Eden Prairie, Minn). Warmed normal saline was administered through an 18-gauge angiocatheter in an ear vein at $30 \mathrm{~mL} / \mathrm{kg} / \mathrm{h}$ for the first hour and $10 \mathrm{~mL} /$ $\mathrm{kg} / \mathrm{h}$ for the duration of the study. Continuous monitoring included heart rate, body temperature, respiratory rate, electrocardiogram, mean arterial pressure, and electroencephalogram (Olympic Medical CFM 6000, San Carlos, Calif). The animals were restrained in dorsal recumbency, and surgical sites were clipped and cleaned with alternating alcohol and 7.5\% povidone-iodine solution. A median sternotomy was performed; the inferior vena cava was encircled with umbilical tape. A 7F pressure-volume conductance catheter (SciSense, London, Ontario) was placed through the left ventricular (LV) apex.

\section{Data Analysis}

Electrocardiogram, heart rate, mean arterial pressure, cardiac output, LV pressure, LV volume, and admittance were sampled at $200 \mathrm{~Hz}$ with a 10-channel analog-to-digital converter (IX/228S Data Acquisition System; iWorx Systems Inc, Dover, NH) and recorded on a computer (MacBook Pro; Apple Computer, Inc, Cupertino, Calif). Instantaneous LV volume was determined by measuring parallel conductance in real-time using the method of Wei and colleagues. ${ }^{16}$ Pressure-volume loops were recorded during a transient cessation in ventilation during the steady-state and inferior vena caval occlusions. Data were analyzed using commercially available software (LabScribe2, iWorx Systems Inc). Preload recruitable stroke work (PRSW) was used as a load-independent index of contractility. ${ }^{17}$ The diastolic relaxation constant tau was calculated using the method of Weiss and colleagues. ${ }^{18}$

\section{Brain Death}

A $14 \mathrm{~F}$ Foley with a $30-\mathrm{mL}$ balloon was inserted into the subdural space through a burr-hole drilled in the left frontoparietal region as previously described. ${ }^{19}$ The balloon was gradually inflated $(1 \mathrm{~mL} / \mathrm{min})$ to $20 \mathrm{~mL}$. Brain death was confirmed by electroencephalographic quiescence and the presence of fixed and dilated pupils. In sham-operated control animals, the scalp was exposed but no catheter was inserted. In animals that became markedly hypotensive in the hours after induction of brain death (mean arterial pressure $<40 \mathrm{~mm} \mathrm{Hg}$ ) unresponsive to volume administration, a bolus of vasopressin ( 0.5 units) was given intravenously and repeated if necessary.

\section{Hemoadsorption Procedure}

Three hours after induction of brain death, animals in the brain death + HA group were connected to an extracorporeal circuit using standard dialysis tubing and a catheter (Arrow International, Reading, Pa) placed in the femoral vein connected to a 300-mL CytoSorb cytokine-adsorption filter. The active component of this device consists of adsorbent beads composed of porous polymerized divinylbenzene. The pores are sized to remove molecules less than $50 \mathrm{kD}$, which is sufficient to remove almost all known cytokines. A peristaltic pump (MasterFlex, Cole-Parmer, Vernon Hills, Ill) was used in conjunction with the circuit. The tubing and filter were primed using approximately $200 \mathrm{~mL}$ of normal saline. Animals were systemically heparinized with 10,000 units of heparin; an additional 5000 units were given every hour to maintain an activated clotting time of 1.5 times baseline. Initial flow through the circuit was set at approximately $340 \mathrm{~mL} / \mathrm{min}$ and adjusted depending on access pressures.

\section{Histology and Myocardial Water Content}

With the use of a number 11 blade, 2 full-thickness biopsies were taken from the LV free wall of each heart just after arrest. One biopsy was fixed in methyl Carnoy's solution, routinely processed, and paraffin-embedded for histologic studies (Histology and Imaging Core, University of Washington Department of Comparative Medicine, Seattle, Wash). Five-micrometer sections were stained with hematoxylin-eosin and Masson's trichrome and then evaluated by a blinded pathologist for stigmata of acute myocardial injury, including glycogenolysis, wavy fibers, and contraction band necrosis. All of the evaluated specimens had areas of myocardium without abnormalities, indicating reasonable tissue preservation.

The other biopsy was blotted dry and weighed on a digital scale to determine wet weight (WW); it was then dried in an oven for several days to 


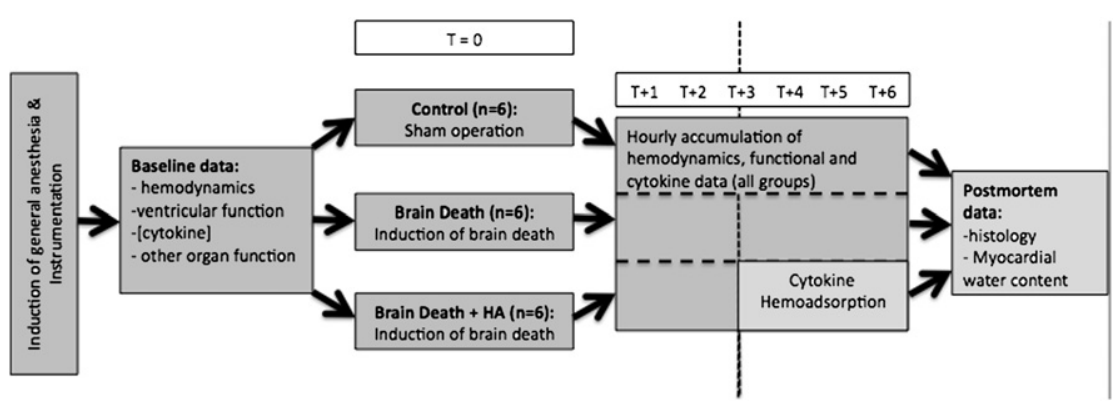

FIGURE 1. Schematic of experimental design. Dashed vertical line indicates initiation of cytokine hemoadsorption (HA) in the brain death + HA group.

a constant weight and reweighed to determine dry weight (DW). Myocardial water content (MWC\%) was calculated as follows:

$$
M W C \%=[(W W-D W) / W W] \times 100
$$

\section{Biochemical Analyses}

Blood samples were drawn into pyrogen-free vials, and plasma was separated by centrifugation and frozen $\left(-80^{\circ} \mathrm{C}\right)$. Serum concentrations of cytokines (TNF, IL-6, and IL-10) were measured at baseline and at hourly intervals for the duration of the experiment. Cytokine concentrations were measured using commercially available enzyme-linked immunosorbent assay kits (R\&D Systems, Minneapolis, Minn), thawing samples only once before using. This assay uses the quantitative sandwich enzyme immunoassay technique. At baseline and at 3 and 6 hours after induction of brain death, blood samples also were drawn to measure aspartate aminotransferase, alanine aminotransferase, blood urea nitrogen, and creatinine, and analyzed by an on-site veterinary laboratory. Plasma neutrophil gelatinase-associated lipocalin (NGAL), a protein expressed in neutrophils and certain epithelia including renal tubules, was used as an early and sensitive biomarker of renal injury ${ }^{20}$ using commercially available enzymelinked immunosorbent assay kits (BioPorto Diagnostics, Gentofte, Denmark).

\section{Statistical Analysis}

Serial data over time within a group were analyzed with repeatedmeasures analysis of variance, and post hoc comparisons were made using the Dunnett test to compare mean values at each time point to baseline values. For comparisons between groups at a given time point, we used 1-way analysis of variance with post hoc comparisons made by Bonferroni's method when appropriate. The primary analysis was the determination of significant differences between groups at 6 hours after brain death (or sham operation); we performed the same analysis at other time points but did not adjust for multiple comparisons. When data did not pass the Kolmogorov-Smirnov normality test for Gaussian distribution or if the Bartlett's test suggested that differences in standard deviations were significant, then the nonparametric Kruskal-Wallis test was used with Dunn's post hoc test where appropriate. Linear regression was performed to determine the correlation between cytokine expression and ventricular function. The Fisher exact test was used to analyze the presence or absence of histologic injury between groups. Analyses were conducted using GraphPad Prism version 5 software (GraphPad Inc, La Jolla, Calif) by an independent biostatistician.

\section{RESULTS}

\section{Baseline Comparisons Between Groups}

There were no significant differences in hemodynamics, indices of ventricular function, or serum markers of renal, hepatic, or pulmonary function at baseline (Tables 1 and 2).
There were no significant differences in any of these parameters between the 2 brain dead groups at the 3-hour mark, confirming that these groups were physiologically equivalent just before the initiation of HA. One animal in the brain death + HA group, approximately 30 minutes after initiating HA, became severely hypotensive despite volume administration and vasopressin; marked right ventricular distension developed, leading to cardiac arrest. Postmortem examination demonstrated diffuse bilateral atelectasis and thrombus formation in both main pulmonary arteries; the animal was included in the study on an intention-to-treat basis. Three animals in each of the brain death groups required a vasopressin bolus for hypotension unresponsive to volume resuscitation.

\section{Effectiveness of Cytokine Hemoadsorption}

Both TNF and IL-6 expression over time were highest in the brain death group, lowest in the control group, and intermediate in the brain death $+\mathrm{HA}$ group (Figure 2, $A$ and $B$ ). This trend was statistically significant for TNF expression beginning 5 hours after the induction of brain death. IL10 expression was detectable only in control animals in the first half of the experiment, so we stopped measuring it.

\section{Effect of Cytokine Hemoadsorption on Systolic Left Ventricular Function}

Systolic LV function as reflected by PRSW is demonstrated by Figure 3, A. For control animals, PRSW was unchanged for the duration of the experiment. In the brain death group, PRSW was significantly reduced versus baseline at 2 hours, whereas in the brain death + HA group, this took 3 hours to occur. After initiating cytokine HA, PRSW leveled off at approximately $80 \%$ of baseline in the brain death + HA group, whereas the brain death group continued to deteriorate: PRSW became significantly worse versus controls at 4 hours after induction of brain death $(67.11 \%$ of baseline vs $105.81 \%$ of baseline, $P=.016)$. At both 5 and 6 hours, PRSW in the brain death group $(50.09 \%$ and $49.79 \%$ of baseline, respectively) was significantly worse than in both the control group $(101.67 \%$ and $102.24 \%$ of baseline, respectively) and the brain death + HA group $(81.78 \%$ and $84.85 \%$ of baseline, respectively, $P<.05)$. $\mathrm{LV}$ maximum change in pressure with time $\left(\mathrm{dP} / \mathrm{dt}_{\max }\right)$, 
TABLE 1. Hemodynamics, ventricular function, and cytokine concentration over time

\begin{tabular}{|c|c|c|c|}
\hline & Baseline & $\begin{array}{c}3 \mathrm{~h} \text { after } \\
\text { brain death }\end{array}$ & $\begin{array}{c}6 \mathrm{~h} \text { after } \\
\text { brain death }\end{array}$ \\
\hline \multicolumn{4}{|l|}{ Heart rate (beats/min) } \\
\hline Control & $82.0 \pm 4.6$ & $80.0 \pm 3.4$ & $89.1 \pm 5.0$ \\
\hline Brain death & $94.0 \pm 8.0$ & $106 \pm 6.7^{*}$ & $101 \pm 11$ \\
\hline Brain death + HA & $76.0 \pm 1.6$ & $96.0 \pm 8.0$ & $111 \pm 11 \dagger$ \\
\hline \multicolumn{4}{|c|}{ Cardiac output (mL/min) } \\
\hline Control & $1732 \pm 355$ & $2748 \pm 630 \dagger$ & $2947 \pm 618 \dagger$ \\
\hline Brain death & $1807 \pm 150$ & $2875 \pm 1060$ & $1221 \pm 239 *$ \\
\hline Brain death $+\mathrm{HA}$ & $1626 \pm 364$ & $2427 \pm 710$ & $1476 \pm 253$ \\
\hline \multicolumn{4}{|l|}{ PRSW (mm Hg) } \\
\hline Control & $50.78 \pm 6.9$ & $51.06 \pm 5.6$ & $51.28 \pm 6.7$ \\
\hline Brain death & $66.28 \pm 3.5$ & $54.35 \pm 4.1$ & $33.88 \pm 7.2 \ddagger$ \\
\hline Brain death $+\mathrm{HA}$ & $62.57 \pm 6.5$ & $\mathbf{4 9 . 0 5} \pm \mathbf{5 . 9} \dagger$ & $53.54 \pm 6.5$ \\
\hline \multicolumn{4}{|l|}{ Tau (ms) } \\
\hline Control & $36.7 \pm 4.2$ & $34.3 \pm 3.0$ & $36.2 \pm 3.5$ \\
\hline Brain death & $36.8 \pm 2.3$ & $43.1 \pm 6.9$ & $62.0 \pm 4.5 \dagger, \ddagger$ \\
\hline Brain death + HA & $41.1 \pm 2.9$ & $38.4 \pm 4.3$ & $37.8 \pm 2.7$ \\
\hline \multicolumn{4}{|l|}{$\mathrm{dP} / \mathrm{dt}_{\max }(\mathrm{mm} \mathrm{Hg} / \mathrm{s})$} \\
\hline Control & $787 \pm 72$ & $786 \pm 94$ & $754 \pm 36$ \\
\hline Brain death & $939 \pm 106$ & $868 \pm 85$ & $893 \pm 136$ \\
\hline Brain death + HA & $831 \pm 81$ & $790 \pm 115$ & $1050 \pm 216$ \\
\hline \multicolumn{4}{|l|}{$\mathrm{dP} / \mathrm{dt}_{\min }(-\mathrm{mm} \mathrm{Hg} / \mathrm{s})$} \\
\hline Control & $1110 \pm 66$ & $1158 \pm 107$ & $1070 \pm 89$ \\
\hline Brain death & $1294 \pm 115$ & $910 \pm 62 \dagger$ & $\mathbf{5 4 5} \pm \mathbf{9 5} \dagger, \ddagger$ \\
\hline Brain death $+\mathrm{HA}$ & $1307 \pm 173$ & $920 \pm 114$ & $1077 \pm 350$ \\
\hline \multicolumn{4}{|l|}{ Ejection fraction (\%) } \\
\hline Control & $32 \pm 4$ & $39 \pm 6$ & $35 \pm 4$ \\
\hline Brain death & $39 \pm 5$ & $33 \pm 4$ & $31 \pm 7$ \\
\hline Brain death $+\mathrm{HA}$ & $37 \pm 4$ & $41 \pm 4$ & $28 \pm 5$ \\
\hline \multicolumn{4}{|c|}{ Mean arterial pressure $(\mathrm{mm} \mathrm{Hg})$} \\
\hline Control & $60 \pm 4$ & $57 \pm 2$ & $56 \pm 3$ \\
\hline Brain death & $58 \pm 3$ & $50 \pm 2$ & $\mathbf{4 5} \pm \mathbf{4} \dagger$ \\
\hline Brain death + HA & $70 \pm 6$ & $52 \pm 5$ & $63 \pm 11$ \\
\hline \multicolumn{4}{|l|}{ IL-6 (pg/mL) } \\
\hline Control & $1.67 \pm 0.76$ & $17.3 \pm 5.3$ & $37.8 \pm 11 \dagger$ \\
\hline Brain death & $4.00 \pm 2.7$ & $34.1 \pm 10$ & $301 \pm \mathbf{1 8 1} \dagger$ \\
\hline Brain death + HA & $3.60 \pm 1.0$ & $22.0 \pm 8.0$ & $94.6 \pm 20 \dagger$ \\
\hline \multicolumn{4}{|l|}{$\mathrm{TNF}(\mathrm{pg} / \mathrm{mL})$} \\
\hline Control & $87.9 \pm 14.9$ & $82.6 \pm 12.8$ & $68.3 \pm 8.55$ \\
\hline Brain death & $60.3 \pm 4.40$ & $95.9 \pm 20.4$ & $106 \pm 13.1^{*}, \dagger$ \\
\hline Brain death + HA & $88.3 \pm 21.9$ & $89.8 \pm 26.9$ & $81.2 \pm 35.2$ \\
\hline
\end{tabular}

Figures are reported \pm standard errors of the mean. Boldface indicates statistically significant results. HA, Hemoadsorption; PRSW, preload recruitable stroke work; $d P / d t_{\max }, \mathrm{LV}$ maximum change in pressure with time; $I L$, interleukin; $T N F$, tumor necrosis factor. $* P<.05$ versus control (same time point). $\dagger P<.05$ versus baseline

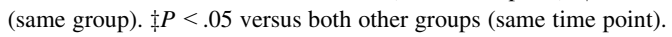

another measure of systolic function, was unchanged over time within each group and unchanged at each time point between groups (Table 1).

\section{Effect of Cytokine Hemoadsorption on Diastolic Left Ventricular Function}

Diastolic LV function as reflected by tau is demonstrated in Figure 3, $B$. There were no significant differences in tau
TABLE 2. Serum markers of noncardiac organ function over time

\begin{tabular}{lccc}
\hline & Baseline & $\begin{array}{c}\text { 3 h after } \\
\text { brain death }\end{array}$ & $\begin{array}{c}\mathbf{6} \text { h after } \\
\text { brain death }\end{array}$ \\
\hline Creatinine $(\mathrm{mg} / \mathrm{dL})$ & & & \\
$\quad$ Control & $0.90 \pm 0.1$ & $1.12 \pm 0.1$ & $1.10 \pm 0.1$ \\
Brain death & $0.98 \pm 0.1$ & $1.42 \pm 0.2$ & $\mathbf{1 . 9 2} \pm \mathbf{0 . 2} *, \dagger$ \\
Brain death + HA & $0.85 \pm 0.1$ & $1.36 \pm 0.2$ & $1.54 \pm 0.2$ \\
BUN/creatinine & & & \\
Control & $9.3 \pm 0.7$ & $9.4 \pm 0.9$ & $12.0 \pm 2.1$ \\
Brain death & $7.7 \pm 0.8$ & $7.0 \pm 0.3$ & $6.4 \pm 0.6$ \\
Brain death + HA & $7.4 \pm 1.4$ & $9.5 \pm 2.0$ & $11.0 \pm 2.4$ \\
NGAL (ng/mL) & & & \\
Control & $145.5 \pm 20.8$ & $137.0 \pm 20.3$ & $163.2 \pm 24.4$ \\
Brain death & $154.1 \pm 14.2$ & $182.9 \pm 21.4$ & $\mathbf{3 1 1 . 2} \pm \mathbf{7 6 . 0} \dagger$ \\
Brain death + HA & $120.7 \pm 16.2$ & $153.6 \pm 28.5$ & $152.2 \pm 38.4$ \\
AST (U/L) & & & \\
Control & $30.3 \pm 4.7$ & $35.7 \pm 1.5$ & $50.0 \pm 7.6$ \\
Brain death & $24.0 \pm 1.2$ & $30.6 \pm 2.2$ & $46.4 \pm 11$ \\
Brain death + HA & $30.3 \pm 5.6$ & $35.8 \pm 6.1$ & $54.8 \pm 12$ \\
ALT (U/L) & & & \\
Control & $32.3 \pm 5.4$ & $28.7 \pm 6.2$ & $28.0 \pm 6.0$ \\
Brain death & $33.0 \pm 1.8$ & $27.8 \pm 2.8$ & $27.6 \pm 2.4$ \\
Brain death + HA & $35.0 \pm 5.6$ & $28.8 \pm 3.7$ & $26.5 \pm 5.5$ \\
Pao $/$ FIo & & & \\
Control & $498 \pm 10$ & $477 \pm 37$ & $443 \pm 48$ \\
Brain death & $543 \pm 25$ & $470 \pm 30$ & $452 \pm 60$ \\
Brain death + HA & $519 \pm 37$ & $442 \pm 57$ & $513 \pm 74$ \\
\hline
\end{tabular}

Figures are reported \pm standard errors of the mean. Boldface indicates statistically significant results. $H A$, Hemoadsorption; $B U N$, blood urea nitrogen; $N G A L$, neutrophil gelatinase-associated lipocalin; $A S T$, aspartate aminotransferase; $A L T$, alanine aminotransferase; $\mathrm{PaO}_{2} / \mathrm{FIO}_{2}$, arterial oxygen tension/inspired oxygen fraction. ${ }^{*} P<.05$ versus control (same time point). $\dagger P<.05$ versus baseline (same group).

over time in the control group or brain death + HA group. However, in brain death, tau became significantly elevated versus baseline at 5 hours and was significantly greater than in both the other groups (and vs baseline) at 6 hours. LV minimum change in pressure with time $\left(\mathrm{dP} / \mathrm{dt}_{\min }\right)$, another measure of diastolic function, showed similar trends: $\mathrm{dP} / \mathrm{dt}_{\min }$ was unchanged over time in both the control and brain death + HA groups, whereas in the brain death group it was significantly diminished versus baseline at 3 hours and significantly reduced versus baseline and versus the other 2 groups at 6 hours (Table 1).

\section{Correlation Between Cytokine Expression and Ventricular Function}

Figure $4, A$ demonstrates the linear regression of TNF concentration as a percentage of baseline versus PRSW as a percentage of baseline. Figure $4, B$ demonstrates the relationship between the log IL- 6 concentration as a percentage of baseline and the PRSW. They both reveal negative slopes that are significantly different from zero but have a loose correlation with $r^{2}$ of 0.3012 and 0.2111 , respectively. The correlation is more tenuous between TNF and IL-6 and tau (Figure 4, $C$ and $D$ ). 


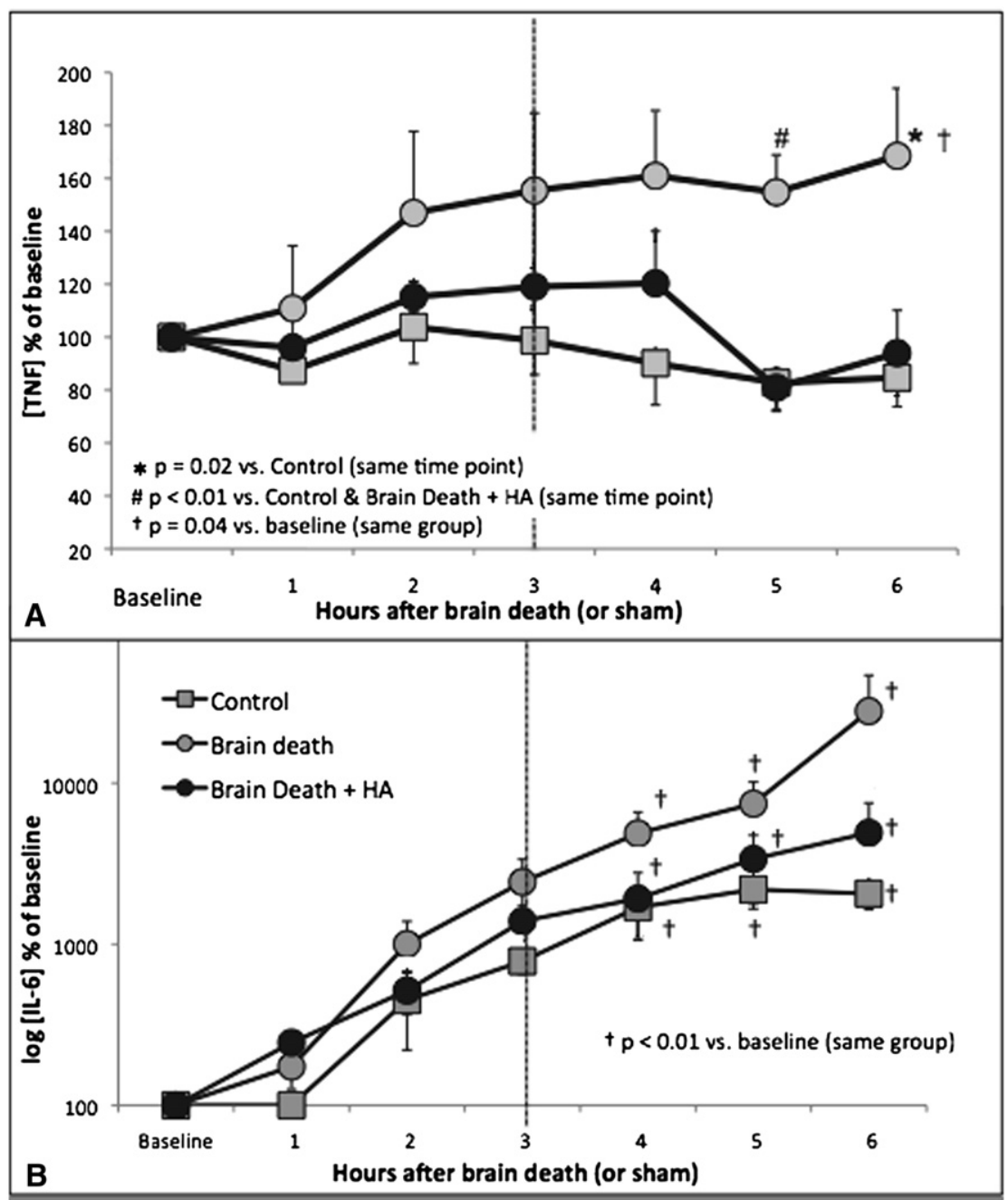

FIGURE 2. Effect of cytokine hemoadsorption (HA) on tumor necrosis factor (TNF) expression (A) and interleukin (IL)-6 expression (B). Brackets indicate standard errors of the mean. Dashed vertical line indicates initiation of cytokine HA in the brain death + HA group.

\section{Other Hemodynamic Indices}

Cardiac output was increased over time versus baseline in the control group, unchanged in the brain death + HA group, and significantly reduced versus control at 6 hours in the brain death group after induction of brain death (Table 1). Mean arterial pressure was unchanged in the control and brain death + HA groups but was significantly diminished in the brain death group at 6 hours. Differences in ejection fraction were not statistically significant between groups.

\section{Effect of Hemoadsorption of Cytokines on Other Organ Function}

Table 2 demonstrates changes in serum markers of renal, hepatic, and pulmonary function over time. Only differences in renal indices were significant, with brain death demonstrating progressively increasing creatinine and NGAL. Doubling of serum creatinine (RIFLE injury) occurred in $0 \%, 67 \%$, and $50 \%$ of animals in the control, brain death, and brain death + HA groups, respectively.

\section{Postmortem Data}

Myocardial water content was significantly greater in the brain death group $(79.2 \% \pm 0.7 \%)$ compared with the control group $(74.1 \% \pm 0.7 \%)$ and brain death + HA group $(75.1 \% \pm 1.07 \%, P<.001)$. Histologic analysis demonstrated contraction band necrosis in 4 of 6 animals in the brain dead group, 2 of 5 animals in the brain dead + HA group, and 1 of 5 animals in the control group. Differences were not statistically significant (Fisher exact test, 2 -sided $P$ value $=.242$ ).

\section{DISCUSSION}

This study demonstrates that extracorporeal, blood purification using CytoSorb HA beginning 3 hours after the induction of brain death attenuates both systolic and 


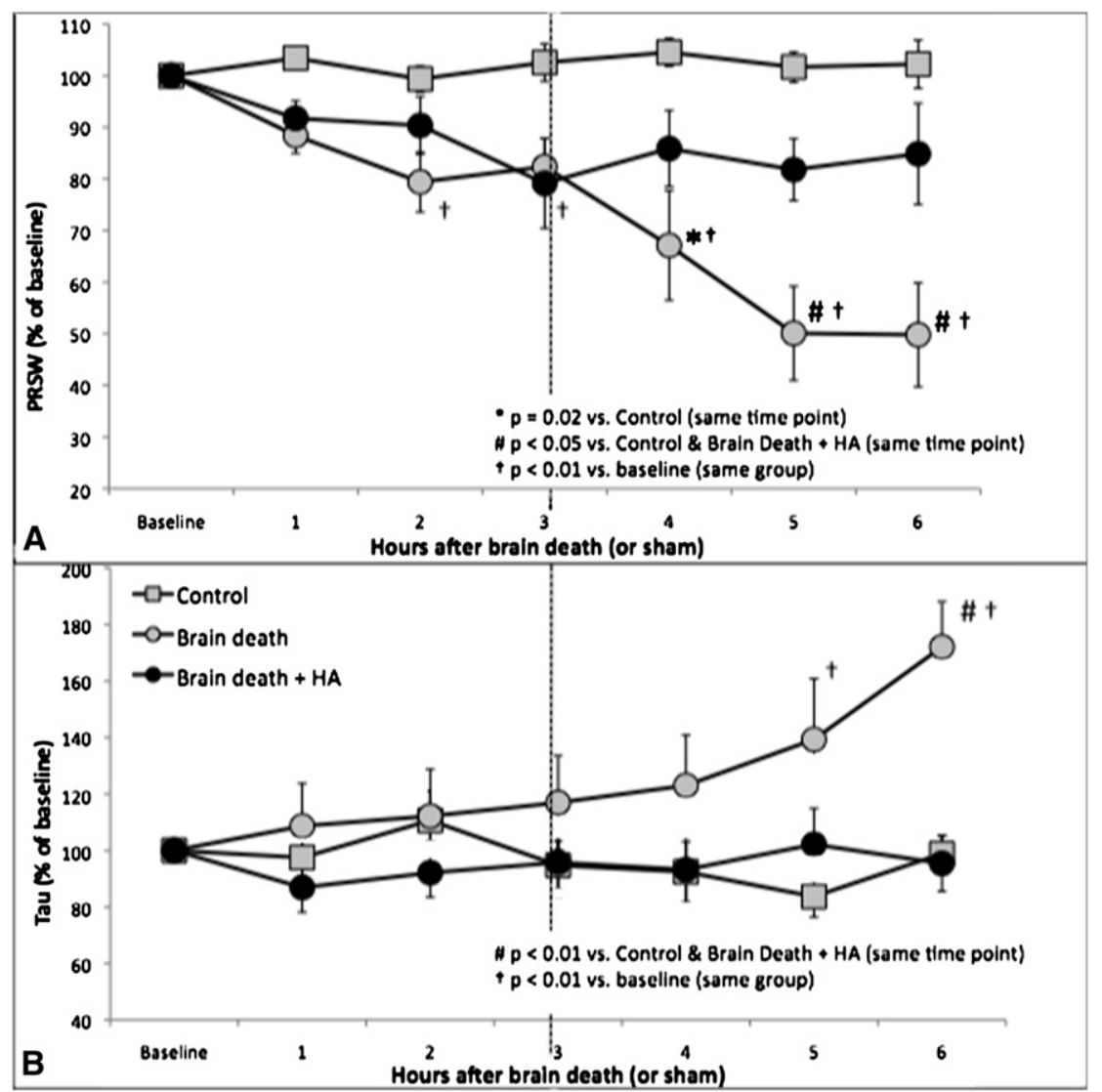

FIGURE 3. Effect of cytokine hemoadsorption $(H A)$ on brain death-induced left ventricular $(L V)$ systolic dysfunction (A) and LV diastolic dysfunction (B). Brackets indicate standard errors of the mean. Dashed vertical line indicates initiation of cytokine HA in the brain death + HA group. PRSW, Preload recruitable stroke work.

diastolic indices of ventricular dysfunction, improves cardiac output and mean arterial blood pressure, and reduces myocardial edema in an open-chest porcine model. Our data from the brain death group are consistent with previous work examining the effect of brain death on the same indices of myocardial performance in pigs both in terms of the extent of dysfunction and the time course of its development. ${ }^{21}$ To our knowledge, this is the first time the CytoSorb HA device has been used in the context of brain death in an experimentally controlled setting where its impact on organ function has been evaluated. The efficacy of cytokine HA appeared more effective for TNF than for IL-6, where the trend in diminished IL- 6 expression in the brain dead treatment group did not reach statistical significance; however, the physiologic response to brain death is notoriously unpredictable, resulting in large standard errors of the mean, thus making demonstrations of differences between groups challenging in a small preliminary study such as this one.

Regression analyses of individual cytokines with individual indices of ventricular function yielded a better albeit loose correlation for systolic indices than for diastolic indices. However, because significant differences in contractility and ventricular compliance were observed between the brain death and the brain death + HA groups at 5 and 6 hours, respectively, after the induction of brain death, and this is the same time frame that significant differences in cytokine expression began to appear between those 2 groups, it is possible that cytokines measured and unmeasured exerted a synergistic effect, as has been demonstrated. ${ }^{22}$ This hypothesis is also supported by recent work in a lowseverity animal model of sepsis in which HA was shown to improve organ function and survival despite a lack of effect of typical cytokines seen in sepsis. ${ }^{23}$ Elements of brain death pathophysiology other than cytokine expression, including hormonal and endocrine derangements, and changes in regional perfusion and loading conditions due to fluctuations in sympathetic neural activity all likely conspired to negatively affect LV function. This is demonstrated by impairment of PRSW in both groups subjected to brain death (Figure 3, $A$, at time points 2 and 3 ) before demonstrable increases in cytokine concentrations; these other factors likely contributed to diluting the correlation between individual cytokine expression and observed changes in function.

The effect of our intervention on systolic function was inconsistent across parameters, with a significant effect 


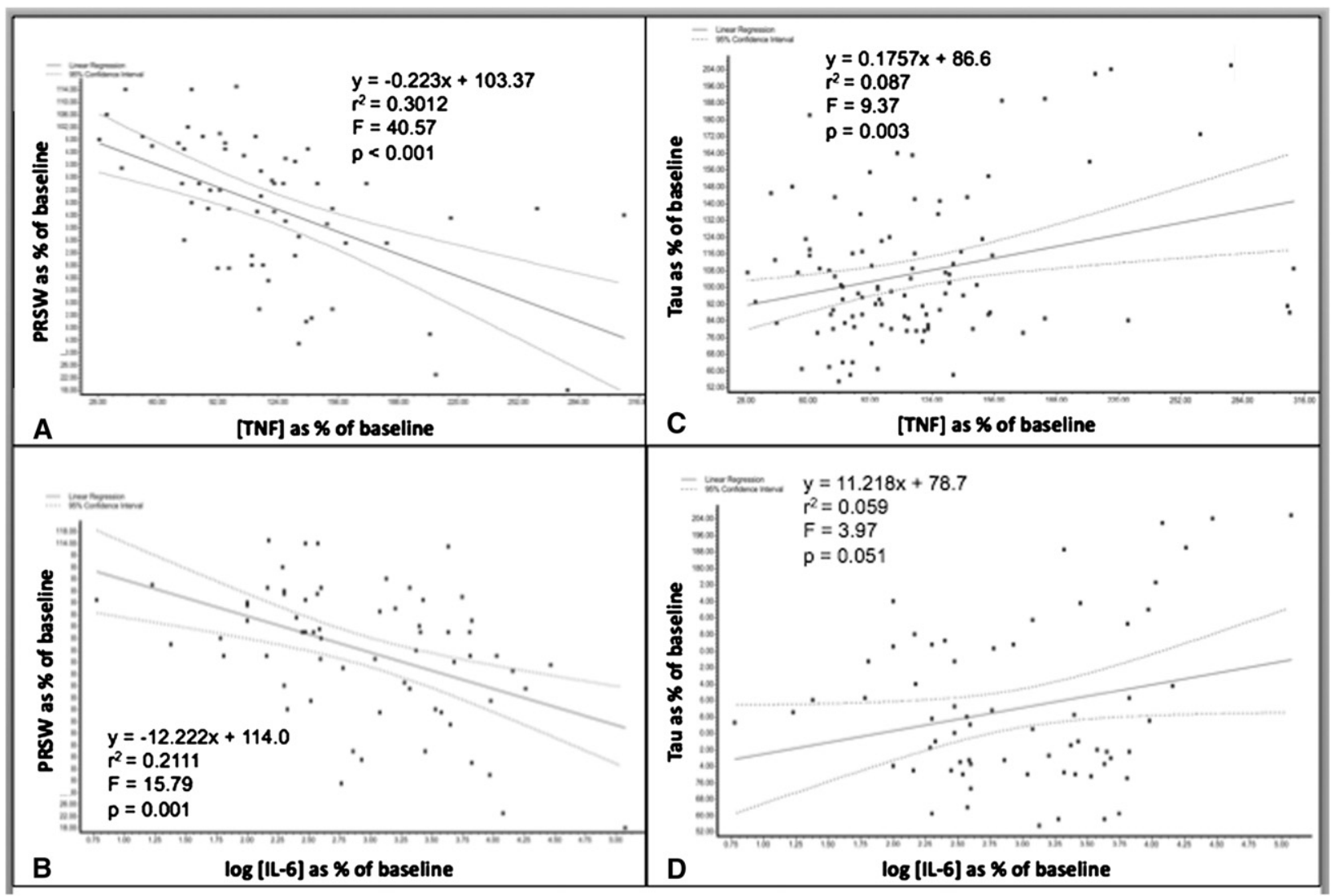

FIGURE 4. Correlation of cytokine concentration with indices of ventricular function. The load-independent index of contractility preload recruitable stroke work $(P R S W)$ is correlated with tumor necrosis factor $[T N F](\mathrm{A})$ and $\log$ interleukin $[I L-6](\mathrm{B})$. The diastolic relaxation constant tau is correlated with $[\mathrm{TNF}](\mathrm{C})$ and $\log$ [IL-6] (D). In all cases, the solid line indicates the linear regression and the dashed lines represent $95 \%$ confidence intervals. The equation for the linear regression, the coefficient of determination, the F statistic, and the $P$ value for the slope of the linear regression are reported for each analysis.

demonstrated when PRSW was evaluated but not when dP/ $\mathrm{dt}_{\max }$ was used. This may be partially explained in that PRSW is a load-independent index of contractility, whereas $\mathrm{dP} / \mathrm{dt}_{\max }$ is not, and therefore potentially confounded by changes in loading conditions known to occur in this setting. The effect on diastolic indices was more consistent with synchronous changes in tau and $\mathrm{dP} / \mathrm{dt}_{\mathrm{min}}$. Significant differences in myocardial edema determined postmortem are also consistent with diminished ventricular compliance. Histologic differences were not statistically significant, and the presence of contraction band necrosis in 1 of the control animals suggests inadequate preservation during the arrest or the presence of preexisting underlying pathology, either of which would confound our pathologic analysis. We were somewhat surprised to observe increases in creatinine, which do not typically occur acutely without pre-renal azotemia (absent in this study); in addition, NGAL is a 20 to $25 \mathrm{kD}$ protein and therefore is within the range of the HA filter (as are the other serum markers measured). Given these concerns, we are reluctant to draw conclusions about the effect of our intervention on renal function despite previous studies that demonstrated a renal-protective effect in a sepsis model. ${ }^{23}$
Although this study did not address the mechanism by which increased cytokine expression might cause ventricular dysfunction, other studies have shown in both the intact $\mathrm{LV}$ and isolated adult cardiac myocytes that alterations in intracellular calcium homeostasis were present during the systolic contraction sequence, resulting in diminished inotropy and correlated with TNF concentration in a dosedependent and reversible fashion; furthermore, this effect was preventable using anti-TNF antibodies. ${ }^{24}$ Other studies in isolated hamster papillary muscles demonstrated that TNF, IL-2, and IL-6 inhibited contractility in a concentration-dependent manner, an effect that was blocked by a nitric oxide synthase inhibitor, suggesting that the negative inotropic effect of these cytokines is mediated through a myocardial nitric oxide synthase. ${ }^{11}$

Our method of attenuating brain death-induced ventricular dysfunction did not result in the same degree of preservation as previous studies that were able to completely prevent deterioration in ventricular function; ${ }^{21,25}$ however, the former study $^{21}$ required administration of a betablocker before the induction of brain death and the latter study $^{25}$ required administration of steroids either before or 
only 1 hour after induction of brain death, requirements that may diminish their clinical relevance.

Potentially confounding aspects of our experiment include the possibility that the CytoSorb device inadvertently absorbed molecules in the same size range as cytokines, such as catecholamines, cortisol, triiodothyronine, and insulin. Although this possibility potentially confounds our ability to isolate the effect of cytokines, it does not impair our evaluation of the overall intervention. Another potential confounder was the lack of a control group using a mock extracorporeal circuit. However, had we used a mock circuit in the brain death group, then differences between the 2 brain dead groups might be attributed to the inflammation resulting from the extracorporeal circuit itself, not the pathophysiology of brain death; we were interested in the impact of the overall intervention because this has the greatest clinical relevance. Therefore, as a result, it is possible that another aspect of the intervention was responsible for the improvements in myocardial performance, for example, it is conceivable that infusion of $200 \mathrm{~mL}$ of normal saline that was used to prime the circuit might have caused increased intravascular oncotic pressure and diminished myocardial edema, which could explain the differences in function. Likewise, it is possible that another aspect of the extracorporeal circuit unrelated to the CytoSorb filter, such as heparin administration, might have affected cytokine expression; therefore, in the absence of a mock circuit, we cannot reliably link observed changes in cardiac function or cytokine expression to the cytokine filter itself, only the overall intervention. Finally, vasopressin administration was used to prevent severe hypotension caused by changes in loading conditions from inducing ischemia and confounding our measurements of myocardial performance; it was used in the same number of animals in the 2 brain dead groups and is unlikely to affect our indices of ventricular function that are load-independent.

\section{CONCLUSIONS}

Although our data offer evidence of a beneficial effect of the overall intervention on myocardial function, an effect that generally correlates with observed differences in cytokine expression, the mechanism of the effect remains unresolved. In addition, the data do not show evidence of improvement in function after initiating HA, only prevention of further deterioration. Consequently, the organ donor population might be a suboptimal application of this therapy because it may be difficult to mobilize the resources to initiate HA therapy in a meaningful time frame. Other potentially more suitable applications might include incorporating the HA filter in the cardiopulmonary bypass circuit for routine or high-risk cardiac surgery procedures to attenuate cardiopulmonary bypass-induced acute organ dysfunction in the cardiac catheterization laboratory immediately after percutaneous revascularization during an acute myocardial event to limit further cytokine-mediated damage or after resuscitation from sudden death when surges in inflammatory cytokines abound.

The authors thank Cytosorbents Inc and Dr Phillip Chan for use of the Cytosorb device, the Department of Comparative Medicine at the University of Washington for help with the animal experiments, and the University of Washington Department of Biostatistics for assistance with the statistical analysis.

\section{References}

1. Pratschke J, Wilhelm MJ, Kusaka M, Basker M, Cooper DKC, Hancock WW, et al. Brain death and its influence on donor organ quality and outcome after transplantation. Transplantation. 1999;67:343-8.

2. Birks EJ, Owen VJ, Burton PBJ, Bishop AE, Banner NR, Khaghani A, et al. Tumor necrosis factor-alpha is expressed in donor heart and predicts right ventricular failure after human heart transplantation. Circulation. 2000;102:326-31.

3. Wilhelm MJ, Pratschke J, Beato F, Taal M, Kusaka M, Hancock WW, et al. Activation of the heart by donor brain death accelerates acute rejection after transplantation. Circulation. 2000;102:2426-33.

4. Pratschke J, Wilhelm MJ, Laskowski I, Kusaka M, Beato F, Tullius SG, et al. Influence of donor brain death on chronic rejection of renal transplants in rats. JAm Soc Nephrol. 2001;12:2474-81.

5. Pandalai PK, McLean KM, Bulcao CF, Duffy JY, D’Souza KM, Merrill WH, et al. Acute $\beta$-blockade prevents myocardial $ß$-adrenergic receptor desensitization and preserves early ventricular function after brain death. J Thorac Cardiovasc Surg. 2008;135:792-8.

6. Galinañes M, Hearse DJ. Brain death-induced impairment of cardiac contractile performance can be reversed by explantation and may not preclude the use of hearts for transplantation. Circ Res. 1992;71:1213-9.

7. Halejcio-Delophont P, Siaghy EM, Devaux Y, Richoux JP, Bischoff N, Carteaux JP, et al. Consequences of brain death on coronary blood flow and myocardial metabolism. Transplant Proc. 1998;30:2840-1.

8. White M, Wieschmann RJ, Roden RL, Hagan MB, Wollmering MM, Port JD, et al. Cardiac B-adrenergic neuroeffector systems in acute myocardial dysfunction related to brain injury; evidence for catecholamine-mediated myocardial damage. Circulation. 1995;92:2183-9.

9. D'Amico TA, Meyers CH, Koutlas TC, Peterseim DS, Sabiston DC, Van Trigt P, et al. Desensitization of myocardial $B$-adrenergic receptors and deterioration of left ventricular function after brain death. J Thorac Cardiovasc Surg. 1995;110:746-51.

10. Tio RA, Nieken J, de Vries EGE, Pfeiffer C, de Jongste MJL, Pieper E, et al. Negative inotropic effects of recombinant interleukin 2 in patients without left ventricular dysfunction. Eur J Heart Fail. 2000;2:167-73.

11. Finkel MS, Oddis CV, Jacob TD, Watkins SC, Hattler BG, Simmons RL. Negative inotropic effects of cytokines on the heart mediated by nitric oxide. Science. 1992;257:387-9.

12. Birks EJ, Burton PBJ, Owen W, Mullen AJ, Hunt D, Banner NR, et al. Elevated tumor necrosis factor-alpha and interleukin-6 in myocardium and serum of malfunctioning donor hearts. Circulation. 2000;102(Suppl III):III-352-8.

13. Song M, Winchester J, Albright RL, Capponi VJ, Choquette MD, Kellum JA. Cytokine removal with a novel adsorbent polymer. Blood Purif. 2004;22:428-34.

14. Kellum JA, Song M, Venkataraman R. Hemoadsorption removes tumor necrosis factor, interleukin-6, and interleukin-10, reduces nuclear factor-kß DNA binding, and improves short-term survival in lethal endotoxemia. Crit Care Med. 2004;32:801-5.

15. Kellum JA, Venkataraman R, Powner D, Elder M, Hergenroeder G, Carter M. Feasibility study of cytokine removal by hemoadsorption in brain-dead humans. Crit Care Med. 2008;36:268-72.

16. Wei C-L, Valvano JW, Feldman MD, Nahrendorf M, Peshock R, Pearce JA. Volume catheter parallel conductance varies between end-systole and end-diastole. IEE Transactions Biomed Engineering. 2007;54:1480-9.

17. Glower DD, Spratt JA, Snow ND, Kabas JS, Davis JW, Olsen CO, et al. Linearity of the Frank-Starling relationship in the intact heart: the concept of preload recruitable stroke work. Circulation. 1985;71:994-9.

18. Weiss JL, Frederiksen JW, Weisfeldt ML. Hemodynamic determinants of the timecourse of fall in canine left ventricular pressure. J Clin Invest. 1976;58:751-60.

19. Purins K, Sedigh A, Molnar C, Jansson L, Korsgren O, Lorant T, et al. Standardized experimental brain death model for studies of intracranial dynamics, organ preservation, and organ transplantation in the pig. Crit Care Med. 2011;39:512-7. 
20. Bangert K, Heslet L, Ghiglione M, Uttenthal LO. NGAL is significantly increased in urine and plasma in acute renal failure. Intensive Care Med. 2006; 32(Suppl 1):S10.

21. McLean KM, Pandalai PK, Pearl JM, Bulcao CG, Lyons JM, Wagner CJ, et al. Beta-adrenergic receptor antagonism preserves myocardial function after brain death in a porcine model. J Heart Lung Transplant. 2007;26:522-8.

22. Cain BS, Meldrum DR, Dinarello CA, Meng X, Joo KS, Banerjee A, et al. Tumor necrosis factor-alpha and interleukin-1 beta synergistically depress human myocardial function. Crit Care Med. 1999;27:1309-18.

23. Peng Z-Y, Wang H-Z, Carter MJ, Dileo MV, Bishop JV, Zhou F-H, et al. Acute removal of common sepsis mediators does not explain the effects of extracorporeal blood purification in experimental sepsis. Kidney Int. 2012;81:363-9.

24. Yokoyama T, Vaca L, Rossen RD, Durante W, Hazarika P, Mann DL. Cellular basis for the negative inotropic effects of tumor necrosis factor-alpha in the adult mammalian heart. J Clin Invest. 1993;92:2303-12.

25. Lyons JM, Pearl JM, McLean KM, Akhter SA, Wagner CJ, Pandalai PK, et al. Glucocorticoid administration reduces cardiac dysfunction after brain death in pigs. J Heart Lung Transplant. 2005;24:2249-54.

\section{Discussion}

Dr Abbas Ardehali (Los Angeles, Calif). This is a relevant and important study in the field of thoracic transplantation. It tries to address the issue of why otherwise healthy donors have poor LV function when the heart is offered for transplantation, and in a broader sense it tries to address the issue of the donor shortage.

To put the issue of donor shortage in perspective, let me share with you some numbers. There were 8100 consented kidney donors last year in the United States, yet only 2300 of them were heart donors, so approximately $70 \%$ of these consented donors could not be heart donors. Although there are many explanations for such discrepancies, such as distance, poor size, match, or donor heart quality (eg, LV hypertrophy or coronary artery disease), an important cohort is a group of donors who are otherwise healthy and have no symptoms, but their heart is just not good. This study tries to address this cohort and suggest that if you attach an extracorporeal circuit to them and remove these "evil humors," the heart is somehow going to turn out to be good and usable. This is a relevant observation in that it can open up a new cohort of potential heart donors.

I want to ask you 3 questions. The first refers to a mechanistic explanation of the beneficial effects of HA. As you mentioned, the filter that you used removes all molecules between 10 and $40 \mathrm{kDa}$, but in addition to cytokines there are many other circulating peptides and nonprotein molecules that are removed or altered via exposure with this membrane. The fact that you just lowered the level of TNF and IL-6 does not necessarily establish a cause-and-effect relationship but just an association. Can you expand on the mechanism of the beneficial effects of HA on the donor heart function?

Dr Rabkin. I could not agree with you more. This filter is not specific to cytokines. In fact, the pores are sized between 20 and $50 \mathrm{kDa}$, but there is actually no way to prevent molecules even smaller than $20 \mathrm{kDa}$ from getting trapped into these pores, so they are not specific to the inflammatory cytokines, and to try to draw a direct link between the observed changes in function and the changes in the cytokines that we noted would be inappropriate. I think we can only say that the overall intervention of placing the animals on the circuit is having some beneficial effect. This study was not designed to investigate the mechanism, but we made some attempts in the study to do various regression analyses to try to draw a link between specific cytokines and specific parameters of ventricular function and found only a loose correlation. I think the highest correlation coefficient was approximately 0.3 , suggesting that $30 \%$ of the observed effect could be attributed to a particular cytokine. There is a lot going on here in brain death physiology other than the surge in cytokines. Certainly those things are contributing to the changes in function that we have seen, but to summarize, to directly address your question, I agree, we cannot attribute the changes here to changes in the cytokine concentration themselves.

Dr Ardehali. The second question is a practical one. As you know, if you plan to do anything to a donor in any hospital in the United States, you have to get the approval from up to 9 other teams procuring other organs in addition to the organ procurement agency. What do we know about the effect of HA on kidneys, livers, lungs, cornea, and skin? The other relevant question is can it be done in a small community hospital? And what are some of the downsides of this technology?

Dr Rabkin. Those are excellent points. In terms of the first question, the effects on other organ function, we looked at some crude measurements of hepatic and renal and pulmonary function. We did not find any changes in hepatic enzymes in any of the groups, neither did we find any changes in the arterial oxygen tension of the animals, which was completely normal for all points after establishment of brain death. In terms of the renal function, we did find that in the 2 brain death groups there was an increase in creatinine over time, and that difference was statistically significant for the brain death group, but we were skeptical there would be such a significant change in creatinine in such a short time frame. Also, in terms of the (RIFLE) criteria, which is a doubling of the baseline creatinine, I think 3 and 4 animals were in the brain death + HA group and the brain death alone group, respectively. Again, we were skeptical of that because in the absence of prerenal azotemia we would not expect to see such a quick increase in creatinine. Finally, we looked at NGAL, which is an early and sensitive biomarker of renal injury, and we were able to show a significant increase in NGAL in the brain death group, which was not present in the brain death + HA group. However, just as I was writing the article I thought to check the size of the NGAL molecule, and it falls squarely in the range that the filter is picking up, so I think you could attribute those differences to the fact that the NGAL was getting caught up in the filter in the experimental group. Therefore, we were not able to show any reliable effects of this technology on other organ function.

In terms of the practicality of doing this and getting agreement from the other teams, logistically it presents a lot of problems. However, it is doable. John Kellum and colleagues at Pittsburgh published an article that demonstrated the feasibility of using this technology in a clinically relevant procurement scenario; they placed brain-dead patients ineligible for organ transplant for whatever reason on the HA circuit and showed that they could bring down the cytokine concentrations and that it was possible to do this. The equipment required is available in a community hospital; I think it is nothing more than you would need for putting patients on dialysis.

The only other comment I would make is that we set out to develop a method of trying to rehabilitate hearts from the pathophysiology of brain death, and that is not really what we finally did. We seemed to arrest the deterioration in function; however, we never 
really showed an improvement, and this is assuming mobilization of the required resources within 3 hours of establishing brain death, which is probably not a realistic time frame in the community to try to get a brain-dead patient into the hospital.
Moderator. David, could you wrap it up please.

Dr Rabkin. Of course, so I am not sure if this is the best application of this technology, but I think it is interesting enough to pursue and might be applicable in other contexts. 